\title{
Initiator and executioner caspases in salivary gland apoptosis of Rhipicephalus haemaphysaloides
}

Yanan Wang ${ }^{1}$, Shanming Hu' ${ }^{1}$, Mayinuer Tuerdi ${ }^{1}$, Xinmao Yu' ${ }^{1}$, Houshuang Zhang ${ }^{1}$, Yongzhi Zhou' ${ }^{1}$ Jie Cao', Itabajara da Silva Vaz Jr. ${ }^{2}$ and Jinlin Zhou ${ }^{1 *}$ (i)

\begin{abstract}
Background: Apoptosis is fundamental in maintaining cell balance in multicellular organisms, and caspases play a crucial role in apoptosis pathways. It is reported that apoptosis plays an important role in tick salivary gland degeneration. Several different caspases have been found in ticks, but the interactions between them are currently unknown. Here, we report three new caspases, isolated from the salivary glands of the tick Rhipicephalus haemaphysaloides.

Methods: The full-length cDNA of the RhCaspases 7, 8 and 9 genes were obtained by transcriptome, and RhCaspases 7, 8 and 9 were expressed in E. coli; after protein purification and immunization in mice, specific polyclonal antibodies (PCAb) were created in response to the recombinant protein. Reverse-transcription quantitative PCR (RT-qPCR) and western blot were used to detect the existence of RhCaspases 7, 8 and 9 in ticks. TUNEL assays were used to determine the apoptosis level in salivary glands at different feeding times after gene silencing. The interaction between RhCaspases 7, 8 and 9 were identified by co-transfection assays.

Results: The transcription of apoptosis-related genes in $R$. haemaphysaloides salivary glands increased significantly after tick engorgement. Three caspase-like molecules containing conserved caspase domains were identified and named RhCaspases 7, 8 and 9. RhCaspase 8 and RhCaspase9 contain a long pro-domain at their N-terminals. An RT-qPCR assay demonstrated that the transcription of these three caspase genes increased significantly during the engorged periods of the tick developmental stages (engorged larval, nymph, and adult female ticks). Transcriptional levels of RhCaspases 7, 8 and 9 in salivary glands increased more significantly than other tissues post-engorgement. RhCaspase9-RNAi treatment significantly inhibited tick feeding. In contrast, knockdown of RhCaspase7 and RhCaspase8 had no influence on tick feeding. Compared to the control group, apoptosis levels were significantly reduced after interfering with RhCaspase 7, 8 and 9 expressions. Co-transfection assays showed RhCaspase7 was cleaved by RhCaspases 8 and 9, demonstrating that RhCaspases 8 and 9 are initiator caspases and RhCaspase7 is an executioner caspase.
\end{abstract}

Conclusions: To the best of our knowledge, this is the first study to identify initiator and executioner caspases in ticks, confirm the interaction among them, and associate caspase activation with tick salivary gland degeneration.

Keywords: Salivary gland degeneration, Transcriptome, Apoptosis, Caspase

\footnotetext{
${ }^{*}$ Correspondence: jinlinzhou@shvri.ac.cn

1 Key Laboratory of Animal Parasitology of Ministry of Agriculture,

Shanghai Veterinary Research Institute, Chinese Academy of Agricultural

Sciences, Shanghai 200241, China

Full list of author information is available at the end of the article
}

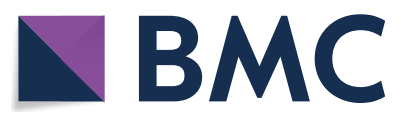

(c) The Author(s) 2020. This article is licensed under a Creative Commons Attribution 4.0 International License, which permits use, sharing, adaptation, distribution and reproduction in any medium or format, as long as you give appropriate credit to the original author(s) and the source, provide a link to the Creative Commons licence, and indicate if changes were made. The images or other third party material in this article are included in the article's Creative Commons licence, unless indicated otherwise in a credit line to the material. If material is not included in the article's Creative Commons licence and your intended use is not permitted by statutory regulation or exceeds the permitted use, you will need to obtain permission directly from the copyright holder. To view a copy of this licence, visit http://creativecommons.org/licenses/by/4.0/. The Creative Commons Public Domain Dedication waiver (http://creativecommons.org/publicdomain/zero/1.0/) applies to the data made available in this article, unless otherwise stated in a credit line to the data. 


\section{Background}

Tick species feed on a wide range of hosts and are important vectors of infectious agents [1-3]. Ticks also may remain attached to the host, feeding on host blood, for long periods [4]. Tick-borne pathogens (TBPs) are transmitted to hosts through tick bites assisted by saliva $[5,6]$.

Programmed cell death (PCD) is a conserved phenomenon, present in eukaryotes and several prokaryotes, and it plays a fundamental role in cell homeostasis [7-9]. PCD is involved in a series of processes and modifications, such as sculpting organs and tissues and removing unwanted structures and damaged cells [10]. Apoptosis is an important component of PCD $[11,12]$. Several studies have attempted to investigate these mechanisms. For example, L'Amoreaux et al. [13] used terminal deoxynucleotidyl transferase dUTP nickend labeling (TUNEL) staining to show that the rate of DNA fragmentation was significantly higher in degenerated salivary glands. In addition, Freitas et al. [14] showed that caspase 3 activity levels increased significantly in tick salivary glands $72 \mathrm{~h}$ post-engorgement.

Caspases are members of the cysteine- 1 aspartatespecific protease family $[15,16]$. There are major groups of caspases in mammals, including initiator caspases and executioner caspases. Caspases 2, 8, 9, 10, 11 and 12 are known to be initiators, and caspases 3,6 and 7 are known to be executioners [17, 18]. Usually, caspases are present in cells as zymogens and require proteolytic cleavage to be converted into active enzymes $[18,19]$. The caspase-associated recruitment domain (CARD) is an interaction motif between different proteins, which plays critical roles in regulating the activation of caspase and nuclear factor $-\kappa B(N F-\kappa B)$ in the context of apoptosis and inflammation [20]. At the beginning of apoptosis, initiator caspases (such as caspase 2 and caspase 9) are activated through the CARD domain and then generate a cascade reaction which activates downstream executioner caspases leading to completion of the apoptosis processes [21]. All caspases are formed by two subunits, a large subunit (p20) and a small subunit (p10). They also have a conserved active-site motif (QACXG; where X is R, Q or $G)$ in the large subunit which mediates the cleavage of proteins at peptide bonds involving the carbonyl group of aspartate residues.

In addition to the well-established role of caspases in apoptosis, there is increasing evidence that caspases have additional functions in several biological events [22]. For example, caspase 1 and caspase 11 play a role in inflammation and in mediating inflammatory cell death caused by pyroptosis [23, 24]. Similarly, caspase 8 plays a dual role in cell death, mediating both receptor-mediated apoptosis and necroptosis. Caspase
3 is involved in tissue differentiation, regeneration, and neural development in a unique manner that does not involve any apoptotic activity [25-28]. Caspase 2, 8 and 14 have demonstrated anti-tumor roles [29, 30]. Caspase 2 is unique with putative roles in maintaining genomic stability, metabolic control, autophagy and aging [31, 32].

Caspases are highly conserved in most organisms, from bacteria to mammals. Although relatively well known in many organisms, information about caspases in ticks is scarce [33-35]. Since the roles of caspases in apoptosis are well known in animals, we postulated they might have a similar role in ticks [33-36]. However, in ticks and tick-borne diseases, the roles of caspases are poorly understood. We used transcriptional analysis to compare tick salivary glands at different feeding times. Transcription of apoptosis-related proteins increased after tick engorgement. Caspase functions were investigated using an RNAi approach in the salivary glands of engorged Rhipicephalus haemaphysaloides.

\section{Methods}

\section{Ticks and tissue collection}

Adult $R$. haemaphysaloides were collected from Wuhan Hubei Province and kept and fed in our laboratory. Different tick developmental stages were attached to the ears of 9-12-week-old female New Zealand White rabbits (SLAC, Shanghai Institutes for Biological Science, CAS, Shanghai, China) using ear bags. After microdissection, tick tissues were washed twice with cold phosphate-buffered saline (PBS, $\mathrm{pH} 7.4$, with $0.14 \mathrm{M} \mathrm{NaCl}$ and $0.0027 \mathrm{M}$ $\mathrm{KCl}, 0.01 \mathrm{M}$ phosphate buffer; Gibco, Life Technologies, Carlsbad, CA, USA) and stored at $-80^{\circ} \mathrm{C}$ in PBS or TRIzol reagent (Invitrogen, Carlsbad, CA, USA).

\section{RNA extraction and CDNA synthesis}

RNA from ticks at different development stages and different tissues from the adult female ticks during different feeding times was isolated using TRIzol reagent (Invitrogen). The synthesis of first-strand cDNA was performed using a HiScript ${ }^{\circledR}$ III RT SuperMix for qPCR (+gDNA wiper) kit (Vazyme Biotech, Nanjing, China) according to the manufacturer's protocol.

\section{Transcriptome of $R$. haemaphysaloides salivary glands}

Salivary glands of both unfed and fed (engorged) $R$. haemaphysaloides females were homogenized in TRIzol reagent (Invitrogen), and purified RNA was used for the construction of paired-end cDNA libraries using a NEBNext ${ }^{\circledR}$ Ultra $^{\text {TM }}$ RNA Library Prep Kit (New England Biolabs, Ipswich, MA, USA), according to the manufacturer's instructions. Sequences were tagged with 
specific barcodes and paired-end reads were sequenced using an Illumina HiSeq platform (Illumina, San Diego, CA, USA) at the Beijing Genomics Institute (BGI, Beijing, China).

RNAseq data were cleaned and formatted using an Agilent 2100 Bioanalyzer (Agilent Technologies, Santa Clara, CA, USA). The high-quality reads were assembled by the Trinity program using default parameters [37]. The assembled transcripts were extended and clustered using TGICL software [38]. The assembled transcripts were processed for further functional annotation and classification analysis. The de novo approach for transcriptome assembly, TransDecoder (http://transdecoder.sourceforg e.net), was used to identify putative CDS sequences from the contigs. Seven different function databases (NR, NT, Gene Ontology (GO) terms [39], Clusters of euKaryotic Orthologous Groups (KOG) [40], Kyoto Encyclopedia of Genes and Genomes (KEGG) pathways [41], SwissPprot and InterPro [42]) were used to annotate all of the assembled transcripts (Unigene). Differentially expressed genes were identified using the MA-plot-based method with a random sampling model by comparing the unfed library to the engorged library. Genes with fold change > 3 and a $P$-value $<0.001$ were considered as differentially expressed genes.

\section{Cloning, sequence analysis and expression of Rhcaspases 7,8 and 9}

Three caspase-like molecules with conserved caspase domains were identified and named RhCaspase 7, 8 and 9. Cloning primers of RhCaspases 7, 8 and 9 (Additional file 1: Table S1) were designed according to ORF regions found in contig sequences assembled in the salivary gland transcriptomes of unfed and engorged $R$. haemaphysaloides. The amplicons with complete ORF sequences were ligated to pMD-18T. SignalP 4.1 (http://www.cbs. dtu.dk/services/SignalP/) [43] and ExPASy (http://web. expasy.org/compute pi/) [44] were used for the signal peptide analysis and isoelectric point (PI) prediction. Similar sequences of target genes were searched using the BLASTp server (National Center for Biotechnology Information, National Institute of Health). The sequences of RhCaspases 7, 8 and 9 were aligned with caspases of other species by Genetyx ver. 6 (Genetyx, Tokyo, Japan). For phylogenetic analysis, the alignment of the sequences was performed using the MUSCLE algorithm [45] and inferred using the maximum likelihood method with the default settings in MEGA X software [46]. Bootstrap support was estimated using 500 bootstrap replicates. The arthropod caspase sequences were obtained from the manually curated database CaspBase [47] and additional tick caspases were obtained from GenBank.
The accession numbers for sequences used are as follows: XM_029966994.1 (Ixodes scapularis caspase 7); XM_029970321.1 (I. scapularis caspase 3); KY056149.1 (Locusta migratoria caspase 9/Dronc). DQ666174.1 (Haemaphysalis longicornis caspase 2); DQ660369.1 (H. longicornis caspase 8); XM_029990833.1 (I. scapularis caspase 3 like); KT194090.1 ( $R$. haemaphysaloides caspase 1); NP_001260718.1 (Drosophila melanogaster Ser/Thr-rich caspase, Dmel $\backslash$ Strica); NP_001303345.1 (D. melanogaster death associated molecule related to Mch2 caspase, Dmel $\backslash$ Damm); NP_476974.1 (D. melanogaster death caspase-1, Dcp-1); NP_477249.3 (D. melanogaster death related ced-3/Nedd2 like caspase; Dredd); NP_477462.1 (D. melanogaster death executioner caspase related to Apopain/Yama, Dmel Decay); NP_524017.1 (D. melanogaster death regulator Nedd2 like caspase, Dmel/Dronc); NP_524551.2 (D. melanogaster death related ICE-like caspase, Dmel/Drice); XP_006570976.2 (Apis mellifera caspase 8); XP_016771440.1 (A. mellifera caspase Dronc); XP_394855.4 (A. mellifera caspase 3); XP_395697.2 (A. mellifera caspase 1); NP_001037050.1 (Bombyx mori caspase 1); NP_001182396.1 (B. mori caspase Nc); NP_001243935.1 (B. mori caspase 4); XP_001648537.2 (Aedes aegypti caspase-1); XP_001655433.2 (Ae. aegypti caspase Dronc); XP_001656809.1 (Ae. aegypti caspase); XP_021694895.1 (Ae. aegypti caspase 7); XP_021704883.1 (Ae. aegypti caspase); XP_021709829.1 (Ae. aegypti caspase 8); XP_021709830.1 (Ae. aegypti caspase 3 isoform); XP_021709833.1 (Ae. aegypti caspase 3); XP_021711617.1 (Ae. aegypti caspase 8); MN_395579 (R. haemaphysaloides caspase 7); ALQ_43547.1 (R. haemaphysaloides caspase 8); and MK_841509 (R. haemaphysaloides caspase 9).

\section{RT-qPCR analyses}

The expression patterns of RhCaspases 7, 8 and 9 were analyzed in ticks feeding during different life stages (larva, engorged-larva, nymph, engorged-nymph, unfed male adult, fed male adult, unfed female adult and engorged female adult). The cDNAs of each stage were used as templates for RT-qPCR, with specific RhCaspase 7, 8 and 9 primers designed using Primer Premier 5 (Additional file 1: Table S2). RT-qPCRs were performed using ChamQ Universal SYBR qPCR Master Mix (Vazyme) green and gene-specific primers with a QuantStudio PCR System (Applied Biosystems, Austin, TX, USA). RT-qPCR cycling parameters were $95^{\circ} \mathrm{C}$ for $30 \mathrm{~s}$, followed by 40 cycles of $95{ }^{\circ} \mathrm{C}$ for $5 \mathrm{~s}$ and $60{ }^{\circ} \mathrm{C}$ for $30 \mathrm{~s}$. All samples were analyzed three times.

The data were normalized to the gene expression of elongation factor-1 (ELF1A, GenBank: AB836665) [48] using the $2^{-\Delta C q}$ method $[49,50] ; \Delta C q$ was calculated by 
subtracting the average ELF1A Cq value from the average $\mathrm{Cq}$ value of the target gene.

\section{Expression of recombinant RhCaspases 7, 8 and 9 and antibody production}

Recombinant RhCaspases 7, 8 and 9 expressed in an Escherichia coli system were used to obtain the sera. Specific RhCaspase 7, 8 and 9 primers were designed for cloning in pET-28a (Additional file 1: Table S3). The RhCaspase 7, 8 and 9 amplified-PCR products were purified and digested with BamHI and HindIII (New England Biolabs) and ligated into pET-28a (Invitrogen) using InFusion HD Cloning Kits (Takara Clontech, Mountain View, CA, USA). Then, BL21 (DE3) (Tiangen, Beijing, China) were transformed with pET-28a-His-RhCaspases 7, 8 and 9. The positive clones were further screened by PCR using specific caspase primers. The expression of RhCaspases 7, 8 and 9 was performed by transforming the pET-28a-His-RhCaspase 7, 8 and 9 recombinant plasmids in $E$. coli BL21 (DE3). Isopropyl $\beta$-D thiogalacto pyranoside (IPTG) was added at a final concentration of 1 $\mathrm{mM}$, and expression was induced at $20{ }^{\circ} \mathrm{C}$ for $12 \mathrm{~h}$. After expression, the recombinant RhCaspases 7, 8 and 9 were affinity-purified under denaturation conditions using His agarose (Merck, Darmstadt, Germany).

For the first injection, 6-8-week-old BALB/c mice (SLAC, Shanghai Institutes for Biological Science, CAS) were immunized with $0.2 \mathrm{ml}$ saline containing $100 \mu \mathrm{g}$ His-RhCaspases 7, 8 and 9 mixed and emulsified with Freund's complete adjuvant (Invitrogen) in an equal volume. For subsequent injections, Freund's incomplete adjuvant (Invitrogen) instead of complete adjuvant was mixed with the same dose of antigen. The mixtures were inoculated 3 times, at 2 -week intervals. Sera were collected three days after the third inoculation. The sera were stored at $-20^{\circ} \mathrm{C}$ until use.

\section{Western blot}

Total proteins of different development tick stages and different tick tissues were extracted using Tris-buffered saline (TBS; $10 \mathrm{mM}$ Tris- $\mathrm{HCl}, \mathrm{pH} 7.5 ; 150 \mathrm{mM} \mathrm{NaCl}$ with $1 \mathrm{mM}$ phenylmethanesulfonyl fluoride). Total extracted protein amounts were determined using the Bradford Protein Assay Kit (Beyotime, Shanghai, China), following the manufacturer's instructions. For SDS-PAGE (12\%; Genescript, Nanjing, China), loading $20 \mu \mathrm{g}$ protein/ well was performed, and proteins on the gel were transferred onto a nitrocellulose membrane. The sera anti His-RhCaspase 7, 8 and 9 were used to detect caspases in protein extracts and an anti-tubulin primary antibody (Proteintech, Rosemont, IL, USA) was used as constitutive control to normalize the signal from the target protein. After primary incubations, the goat anti-mouse IgG $(\mathrm{H}+\mathrm{L})$ secondary antibody conjugated with HRP (Invitrogen), and IRDye 800CW goat anti-mouse IgG (H + L) (LI-COR, Nebraska, USA) were used as secondary antibodies in assays. Images were captured by ChemiDoc Touch (Bio-rad, Hercules, CA, USA) or Odyssey Imaging System (LI-COR).

\section{RNAi of Rhcaspases 7, 8 and 9}

The RNAi experiments were designed against RhCaspase 7, 8 and 9 genes. For the design of RNAi primers, caspase sequences were screened by Primer Premier 5. Caspasespecific primers (Additional file 1: Table S4) containing the T7 polymerase promoter sequence were used for PCR amplification. The amplicons were then purified to obtain templates for double-stranded RNA synthesis using the T7 RiboMAX Express RNAi system (Promega, Madison, WI, USA). Unfed female ticks were microinjected with approximately $1 \mu \mathrm{g}$ of dsRNA caspase. Control ticks were injected with unrelated dsLuciferase. The biological parameters analyzed were: attachment rate at $48 \mathrm{~h}$; number of engorged ticks; and weight. RT-qPCR was used to evaluate gene silencing efficiency.

\section{TUNEL staining}

Dissected salivary glands were fixed in $4 \%$ formalin and embedded in paraffin. Sections of salivary glands were mounted on microscope slides. Tissue sections were then deparaffinized, washed in 100\% ethanol, and rehydrated. Samples were washed with PBS. After antigen retrieval with $0.1 \%$ Triton $\mathrm{X}-100$, the tissues were incubated for $1 \mathrm{~h}$ with 1:9 TdT mixed with fluorescent-labeled dUTP at $37{ }^{\circ} \mathrm{C}$, following the instructions of the Roche in situ Cell Death Detection Kit, POD (Roche, Mannheim, Germany). The cell nuclei were stained with $1 \mu \mathrm{g} / \mathrm{ml} 4$, 6'-diamidino-2-phenylindole (DAPI; Invitrogen) in distilled $\mathrm{H}_{2} \mathrm{O}$ for $20 \mathrm{~min}$. After washing, the sections were mounted using Lab Vision ${ }^{\mathrm{TM}}$ PermaFluor $^{\mathrm{TM}}$ (Invitrogen) medium under glass coverslips, then viewed and photographed on a Pannoramic DESK Digital Slide Scanner (3D Histech, Budapest, Hungary).

\section{Cells and transient co-transfection assays}

HEK 293 cells were maintained in Dulbecco's modified Eagle's medium (DMEM; Gibco), supplemented with $8 \%$ heat-inactivated fetal bovine serum (Biological Industries, Kibbutz Beit Haemek, Israel) and $1 \%$ penicillin (Gibco) at $37^{\circ} \mathrm{C}$.

The full-length ORF of RhCaspases 7, 8 and 9 were inserted into the $\mathrm{p} 3 \times$ Flag-CMV-14 vector (MiaoLing Plasmid Sharing Platform, Wuhan, China) with Flag tag at the N-terminal with gene-specific primers (Additional 
file 1: Table S3). Transfection using Lipofectamine ${ }^{\mathrm{TM}}$ 3000 Transfection Reagent (Invitrogen) was performed according to the manufacturer's protocol with a DNA to Lipofectamine ratio of 1:2w/v. The HEK 293 cells were transformed with $3 \mu \mathrm{g} /$ well of plasmid or co-transfected with the equivalent amount of two different plasmids in 6-well plates.

\section{Data analysis}

GraphPad PRISM 6.0 software (Graph Pad Software Inc., San Dieo, CA, USA) was used for all data analyses. Mean \pm standard error (SE) values were calculated for three independent experiments, and two-tailed Student's t-tests were used to identify significant differences between groups $\left({ }^{*} P<0.05,{ }^{* *} P<0.01\right.$, ${ }^{* * * *} P<0.001$, ${ }^{* * * *} P$ $<0.0001)$.

\section{Results}

Transcriptome analysis of tick salivary glands

Unfed and engorged tick salivary glands were selected for transcriptome analysis and used to observe the expression of apoptosis-related genes. After RNAseq analyses, 65.38 and $44.16 \mathrm{Mb}$ clean reads were obtained from unfed and engorged tick salivary glands, respectively. Reads were assembled and 39276 and 34725 unigenes were annotated from salivary glands of unfed and engorged ticks (Fig. 1a).

Using unfed tick salivary glands as a reference, 6825 and 9994 unique genes were upregulated and downregulated, respectively, in engorged tick salivary glands (Fig. 1b). The majority of the genes were expressed with $\log 2$ fold change values $>2$ and $\leq 10$ (about 95\%). Genes showing values $>10$ and $\leq 20$ were less abundant (about 5\%) (Fig. 1b). We deposited the RNAseq raw data to the NCBI SRA repository (accession numbers: SRR11523177 and SRR11523176). The enrichment GO terms (Additional file 2: Figure S1), Pheatmap-plot (Additional file 2: Figure S2), FASTA format sequence of unigenes (Additional file 3: Alignment S1) and differentially expressed genes lists (Additional file 4: Dataset S1) are provided as additional files.

\section{Both intrinsic and extrinsic apoptosis pathways were activated during tick salivary gland degeneration}

Caspase-dependent apoptosis pathways are divided into extrinsic and intrinsic pathways. Based on known sequences in other organisms, the RNAseq analysis of the tick salivary gland identified 28 apoptosis-related genes; 10 were related to the extrinsic apoptotic signaling pathway; and 11 were related to intrinsic apoptotic signaling pathway. Three were involved in the apoptosis execution phase and nine other genes were involved in apoptosis (Fig. 2a-d). Most of the apoptosis-related genes were upregulated after engorgement (Fig. 2a-d). These results suggest that apoptosis pathways have an important role in the degeneration of tick salivary glands. Based on the molecular analysis and expression profile, some representative components of apoptosis pathways a

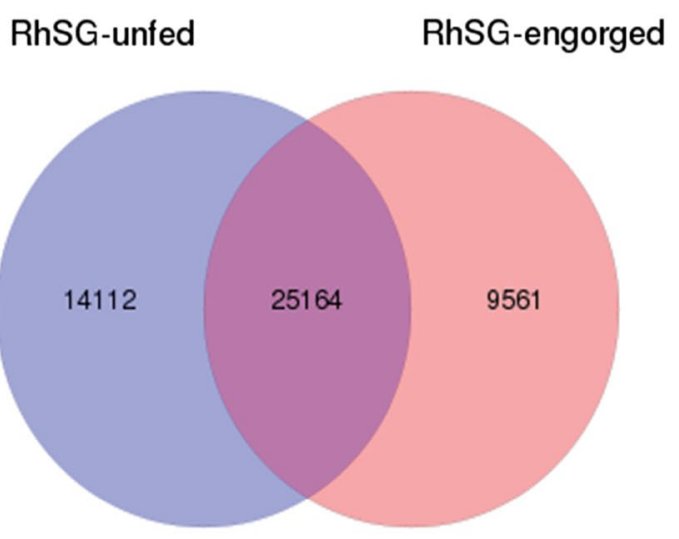

b MA plot for RhSG-unfed vs RhSG-engorged

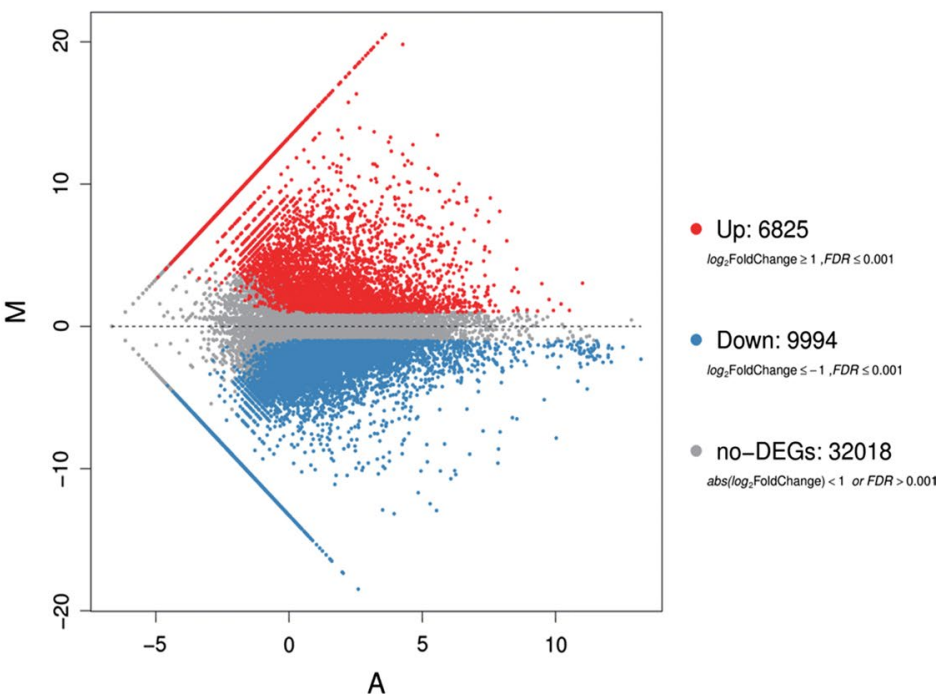

Fig. 1 Transcriptome sequencing and differentially expressed gene analysis of unfed and engorged Rhipicephalus haemaphysaloides salivary glands. a Venn analysis of the transcriptome sequencing of unfed and engorged tick salivary glands. b Total number of differentially expressed genes (upregulated and downregulated) between unfed and engorged tick salivary glands 


\section{a}

\section{Extrinsic}

fatty acid synthase(FAS)

fatty acid synthase(FAS)

fatty acid synthase(FAS)

fatty acid synthase(FAS)

fatty acid synthase(FAS)

fatty acid synthase(FAS)

fatty acid synthase(FAS)

fatty acid synthase(FAS)

fatty acid synthase(FAS)

TNF alpha induced protein 1

\section{GeneID}

CL1190.Contig2_Al

Unigene20377 All

Unigene23136_All

CL3723.Contig4_All

CL2339.Contig2_Al

Unigene20302_All

Unigene20497_All

Unigene24606_All

Unigene20239_All

Unigene5599_All

C

\section{Execution}

caspase-7 like

poly [ADP-ribose] polymerase

poly [ADP-ribose] polymerase-like

\section{GenelD}

CL3173.Contig3 A

Unigene13526_All

Unigene16939 All

\section{b}

d

HtrA serine peptidase 1

caspase-2 like

proteinral transcription factor BTF3

Inhibitor of apoptosis-promoting Bax1

Diablo homolog, mitochondrial

\section{GenelD \\ Unigene21915_All CL1492.Contig1_Al Unigene19204_All CL4607.Contig1_All Unigene5392_All \\ Unigene10686_All Unigene17326_All CL3060.Contig1_All Unigene18469_All Unigene5599_All \\ CL443.Contig1_All

All

All

.

Fig. 2 Expression of apoptosis related genes in Rhipicephalus haemaphysaloides salivary glands upregulated post-engorgement. a Intrinsic apoptosis pathway. b Extrinsic apoptosis pathway. c Executioner components. d Other apoptosis related genes. Light green, upregulated genes were selected as those between $2-10$ fold $(1<\log 2$ normalized fold change $<3.32)$; green, upregulated genes were selected as those expressed by more than 10 fold (log2 normalized fold change $>3.32$ ); Pink, downregulated expressed gene were selected as those with a $2-10$ fold $(2<$ log 2 normalized fold change < 3.32); Red, downregulated expressed gene were those with a more than 10 fold (log2 normalized fold change > 3.32)

were selected to characterize the tick salivary gland degeneration process.

\section{Identification of RhCaspase 7, 8 and 9}

Using nucleic acid sequences obtained from RNAseq, specific cloning primers were designed according to the predicted sequences of $R$. haemaphysaloides caspase 7, 8 and 9. The ORF regions of the three caspase-related genes were cloned from the cDNA of fully engorged $R$. haemaphysaloides salivary glands and named RhCaspase 7, 8 and 9. RhCaspase7 ORF (GenBank: MN395579) has 903 bp encoding a protein of 301 amino acid residues (Fig. 3a, b) with a deduced molecular weight (MW) and theoretical isoelectric point (PI) of $34 \mathrm{kDa}$ and 6.39, respectively. RhCaspase8 (GenBank: ALQ43547.1) and RhCaspase9 ORF (GenBank: MK841509) were also cloned, with 1368 bp and $1164 \mathrm{bp}$, respectively, and encoding proteins of 456 and 388 amino acid residues, respectively (Fig. 3a, b). They have deduced MWs of $51.7 \mathrm{kDa}$ and $40.4 \mathrm{kDa}$, and PIs of 6.97 and 6.5, respectively. Sequence and structure analysis showed that they have similarity to mammal caspases. RhCaspase7, RhCaspase8, and RhCaspase9 had the conserved active site (QACR (I) G), a large subunit (p20 domain), and a small subunit (p10 domain), respectively. Similar to the executioner caspases, RhCaspase7 has a short prodomain in the N-terminus. In contrast, as in other initiator caspases, both RhCaspase 8 and RhCaspase9 have a long N-terminal pro-domain (more than 90 amino acids). The pro-domain of RhCaspase8 named with MoCF-biosyth (involved in biosynthesis of molybdopterin cofactor). The structure of this domain is known, and it forms an $\alpha / \beta$ structure. In the known structure of gephyrin this domain mediates trimerisation. RhCaspase9 has a low complexity domain in the N-terminal, which is a molecular structural region of biased composition (Fig. 3a).

The phylogenetic analysis showed the relationship between tick caspases and caspases of other arthropods. Caspases 8 and 9 branched into one clade and caspase 7 branched to a different clade (Fig. 3c). All R. haemaphysaloides caspases grouped with similar arthropod caspases (Fig. 3c, Additional file 1: Figure S3).

\section{Expression of RhCaspases 7, 8 and 9 and production of antisera}

The coding sequences of RhCaspases 7, 8 and 9 were cloned into prokaryotic expression vectors (pET-28a) to produce recombinant RhCaspases 7, 8 and 9. All of the recombinant proteins were expressed as inclusion bodies in $E$. coli. The recombinant proteins undergo a 


\section{a}

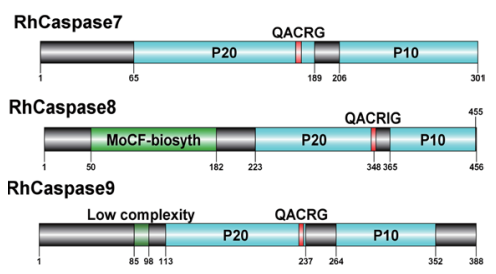

b

RhCaspase7

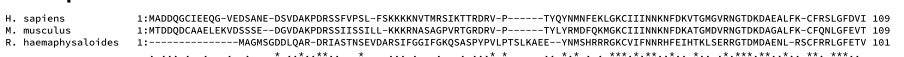

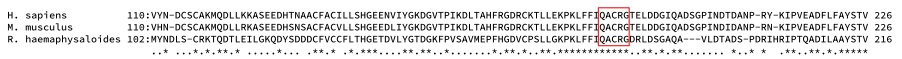

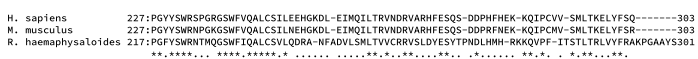

RhCaspase8

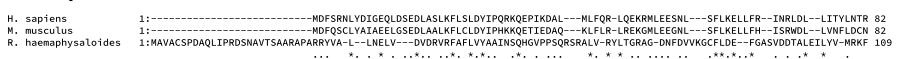

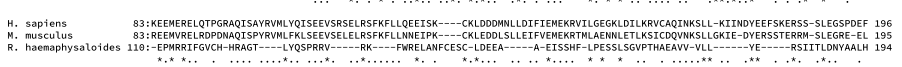

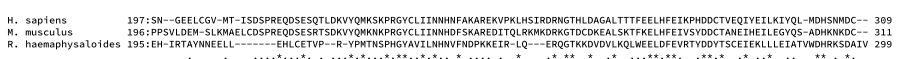

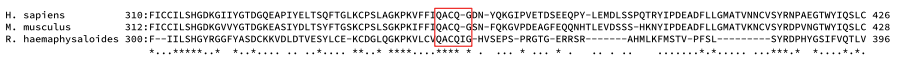

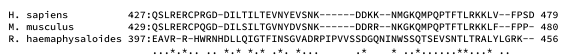

RhCaspase9

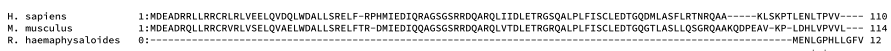

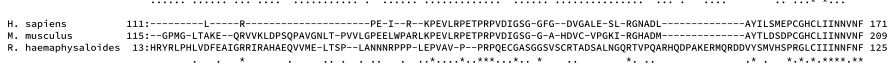

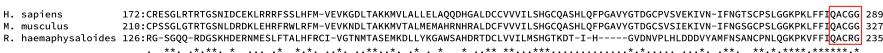

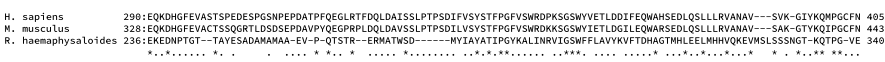

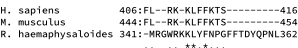

Fig. 3 Sequence and structure analysis of Rhipicephalus haemaphysaloides RhCaspases 7, 8 and 9. a Domain structure of RhCaspases 7, 8 and 9. b Alignment of the deduced amino acid sequences of RhCaspases 7, 8 and 9. Homo sapiens caspase 7: P55210; Mus musculus caspase 7: P97864; Homo sapiens caspase 8: Q14790; Mus musculus caspase 8: O89110; Homo sapiens caspase 9: P55211; Mus musculus caspase 9: Q8C3Q9. c Phylogenetic tree of $R$. haemaphysaloides caspases with the caspases of other arthropod species. Bootstrap values after 500 simulations are shown at the branches and RhCaspases 7,8 and 9 are marked with red rectangles

spontaneous cleavage as previously observed in recombinant caspases of Spodoptera frugiperda and B. mori [51, 52].

After solubilisation and purification, His-RhCaspases 7, 8 and 9 (molecular weight: $\sim 41 \mathrm{kDa}, \sim 58.5 \mathrm{kDa}$ and $\sim 47.3 \mathrm{kDa}$ were obtained, respectively (Fig. $4 \mathrm{a}-\mathrm{c}$ ). Initiator caspases had two cleavage sites, one located between the pro-domain and p20 and another between p20 and p10 (Fig. 4b, c). The MWs of proRhCaspases and cleaved-RhCaspases were similar to the corresponding mammalian caspase molecules. Purified recombinant RhCaspases 7, 8 and 9 were used to elicit polyclonal antibodies in rabbits. Western blot analysis (Fig. $4 \mathrm{~d}-\mathrm{f}$ ) revealed that the three sera were able to identify the full c

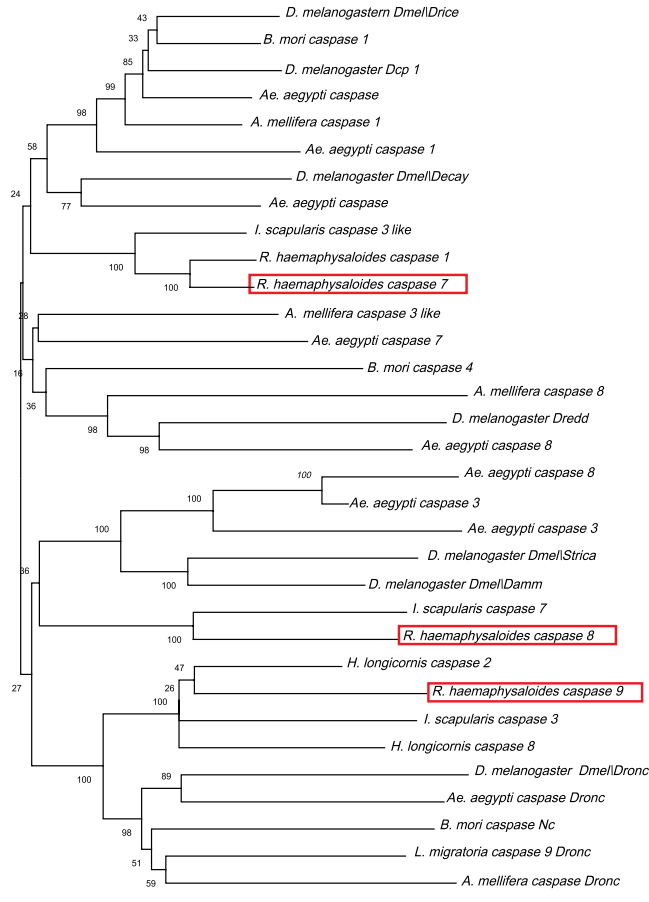

0.20 recombinant proteins and the cleavage forms, and the fragment sizes were consistent with those observed by SDS-PAGE (Fig. 4a-f).

\section{Transcription and translation of caspase profiles in different stages, tissues and feeding periods}

The cDNA of eggs, larvae (unfed and engorged), and nymphs (unfed and engorged) were subjected to RTqPCR to evaluate the expression profiles of RhCaspase 7, 8 and 9 genes during the developmental stages (Fig. 5a). The cDNA of unfed adults (female and male), fed adults (female and male), and engorged female ticks were used to determine the sex-specific profiles of RhCaspase 7, 8 and 9 genes. The transcription level of 


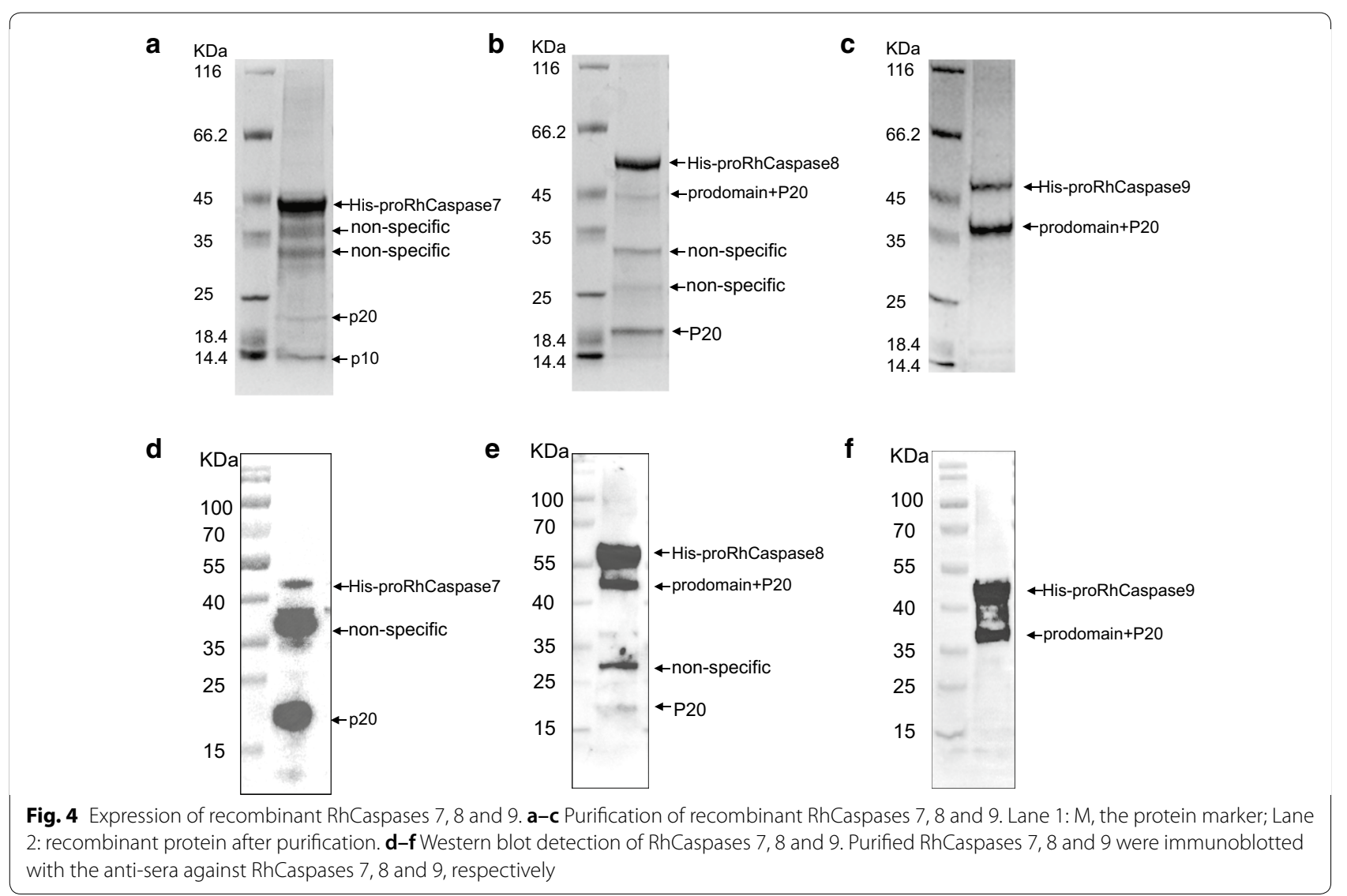

RhCaspases 7, 8 and 9 increased after feeding (Fig. 5a). The expression of RhCaspase 7, 8 and 9 genes did not change in male ticks at different feeding times, but in female ticks the expression level of RhCaspase 7, 8 and 9 genes increased during feeding (Fig. 5b).

After microdissection, the cDNA of salivary glands, ovary and midgut of adult female ticks at different feeding times were analyzed for RhCaspase 7, 8 and 9 gene expression profiles. RhCaspase 7, 8 and 9 genes are expressed in different tissues during tick feeding (including the early feeding period, the fast feeding period, and the end of the feeding period). However, the different genes had different expression levels in different organs. The expression levels of the RhCaspase 7 gene in the salivary gland and ovary were higher than in the midgut. Similar to levels in the salivary gland of fully engorged ticks after feeding, the RhCaspase 9 gene had consistent high expression in the midgut during all feeding time periods. However, the RhCaspase 8 gene had a low expression level in all three tissues (Fig. 5ce). The expression of the three RhCaspases increased in the salivary gland during feeding, which supports the role of apoptosis in the process of salivary gland degeneration (Fig. 5c).
Western blot analysis using anti-caspases sera showed that, in the salivary gland, caspases were cleaved during the feeding process. This is associated with salivary gland degeneration. Cleaved-RhCaspases 7 and 8 were detected in the fast feeding time (fed for 5 days to fed for 7 days) to post-engorgement and cleaved-RhCaspase 9 was detected after day 3 (Fig. 6). Caspase fragments matched the size of putative caspase fragments calculated by in silico analysis.

\section{RNAi of RhCaspases 7, 8 and 9}

To identify function of RhCaspases 7, 8 and 9, the genes of RhCaspases 7, 8 and 9 were targets of RNA interference, in vivo. qRT-PCR analysis of ticks showed decreased levels of caspase mRNAs in caspase-dsRNA injected groups compared to luciferase-dsRNA injected controls (Fig. 7b) (RhCaspase 7 RNAi: $t_{(3)}=11.08, P$ $=0.0004$; RhCaspase 8 RNAi: $t_{(3)}=33.64, P<0.0001$; RhCaspase 9 RNAi: $\left.t_{(3)}=15.16, P=0.0001\right)$. Compared to the control groups, ticks injected with RhCaspase 9-dsRNA and RhCaspase 8-dsRNA showed a reduction in size on the sixth day after treatment and the effect was most obvious in the RhCaspase 9-dsRNA group (Fig. 7a). Consequently, the engorged weight of the caspase 


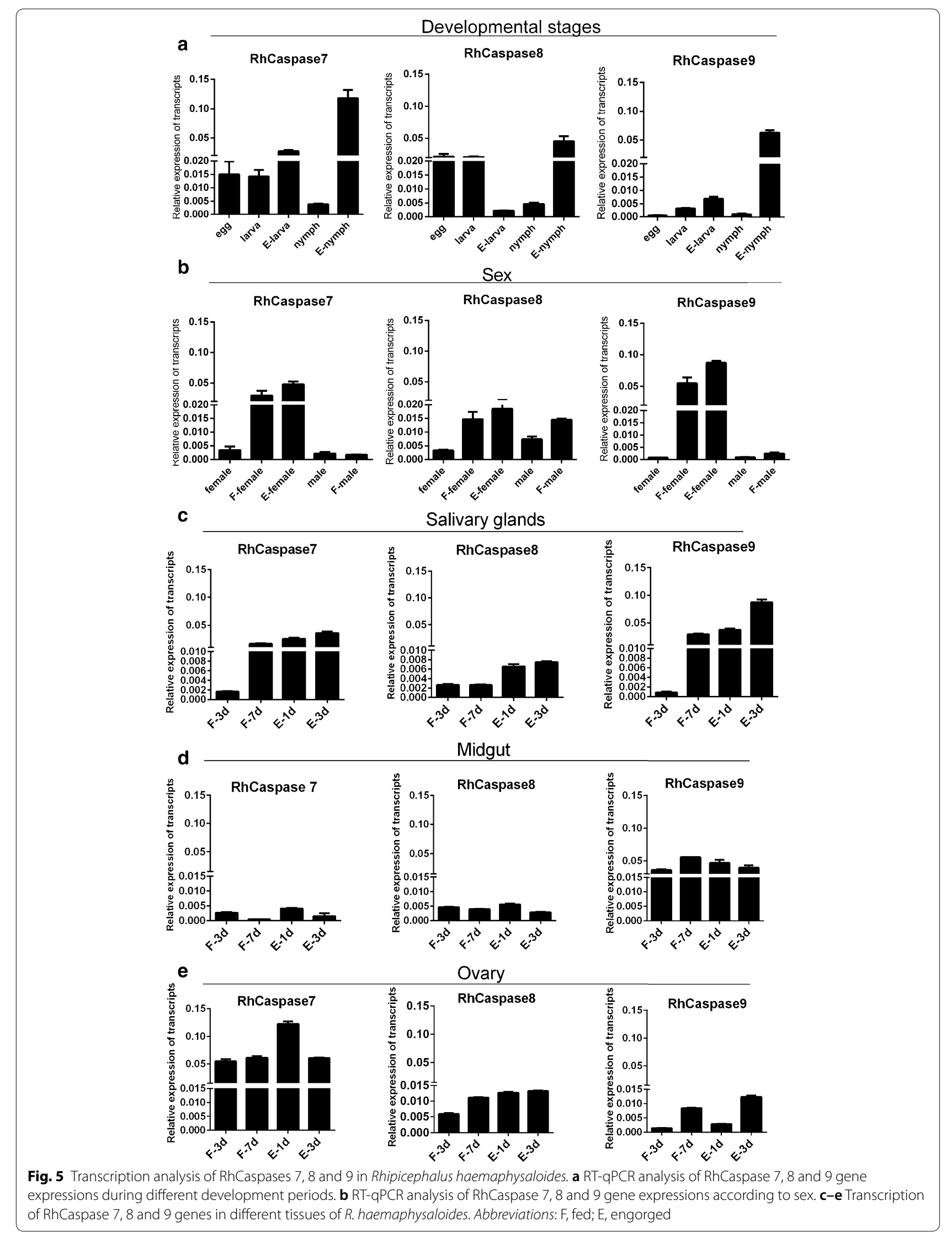




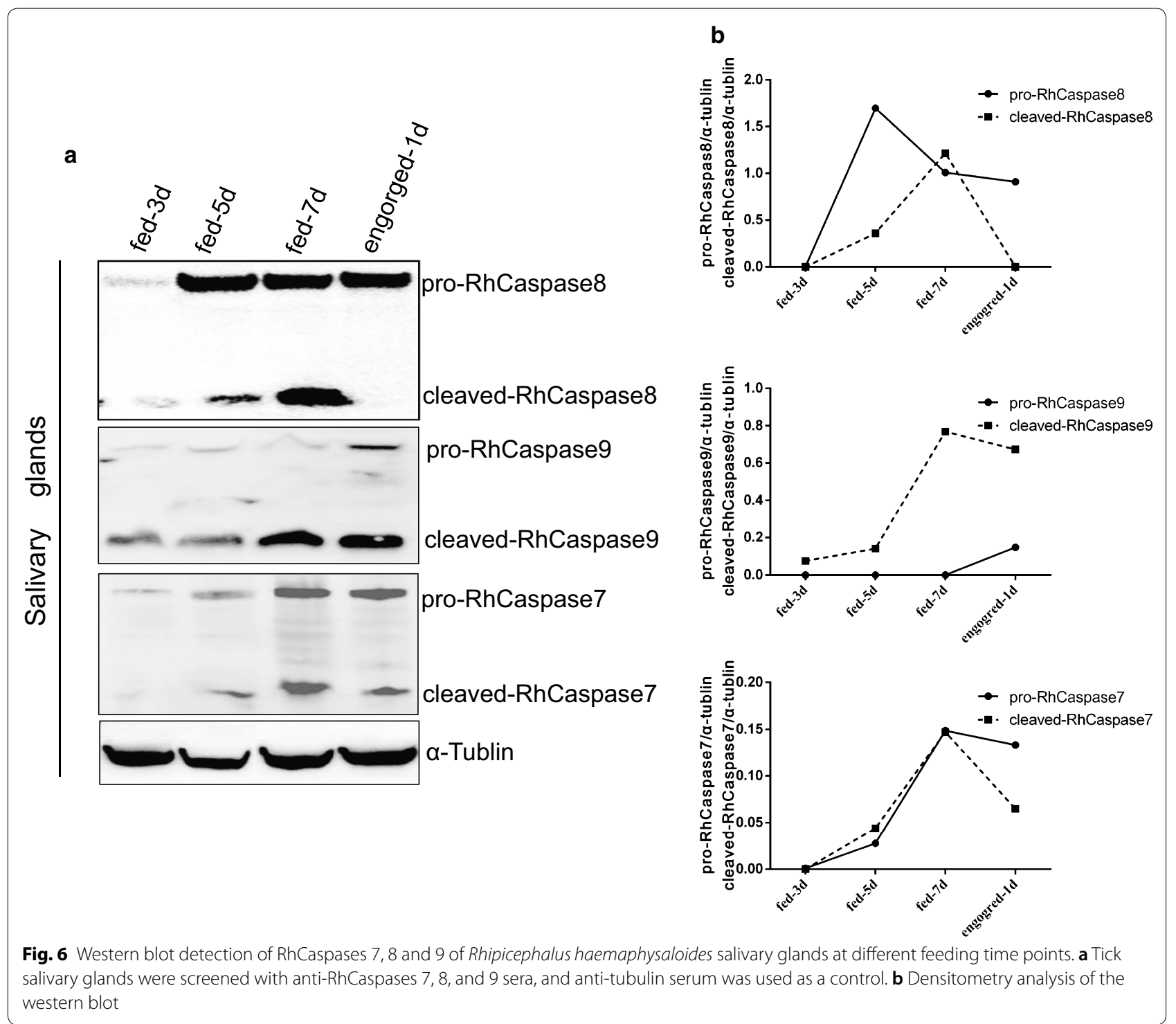

9-dsRNA injected group was significantly lower than the control (Table 1). However, there was no significant difference in the attachment rate between the different experiment groups and control groups.

TUNEL assays showed that the salivary glands of caspase-dsRNA injected groups (RhCaspases 7, 8 and 9) had reduced apoptosis levels compared with the control group (Fig. 7c).
RhCaspase 7 can be cleaved by RhCaspases 8 and 9 in vitro Co-transfection of HEK 293 cells with plasmids containing two different RhCaspase genes produced coexpression of the proteins. The results also showed that RhCaspase-7 is cleaved by the other two RhCaspases (Fig. 8). This confirms the in silico prediction that RhCaspase 7 is an executioner caspase and RhCaspases 8 and 9 are initiator caspases.

(See figure on next page.)

Fig. 7 Biological effects of RNAi of Rhipicephalus haemaphysaloides RhCaspases 7, 8 and 9 in vivo. a Images comparing ticks inoculated with dsRhCaspases 7, 8 and 9 with control groups on day 6 of feeding. dsRhCaspase 9 inhibited the blood-feeding process and resulted in ticks smaller than the controls. $\mathbf{b}$ Confirmation of RhCaspase 7,8 and 9 silencing using RT-qPCR. Total RNA was extracted on feeding day 5 from female ticks injected with dsRNA. Bars represent mean relative expression of RhCaspase 7, 8 and 9 genes; error bars represent standard error. ${ }^{* * *} P<0.001$, based on two-tailed Student's t-tests. c Positive TUNEL staining decreased after RNAi of RhCaspases 7, 8 and 9, and DNA was stained with DAPI. Scale-bars: c, $50 \mu \mathrm{m}$ 
a

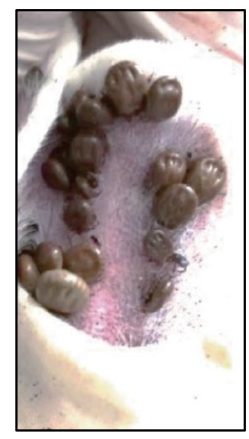

dsRhCaspase7

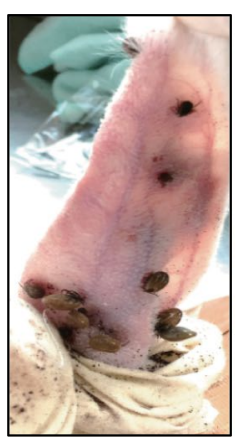

dsRhCaspase8

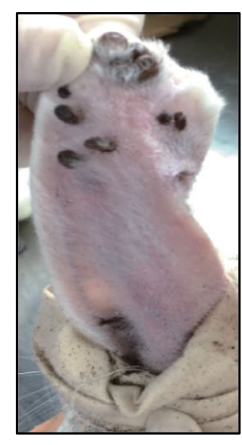

dsRhCaspase9

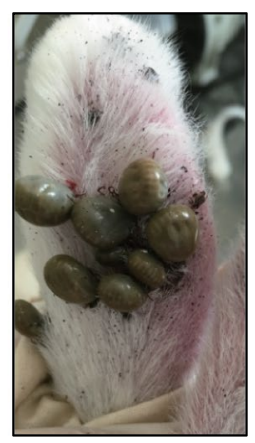

dsLuciferase

b
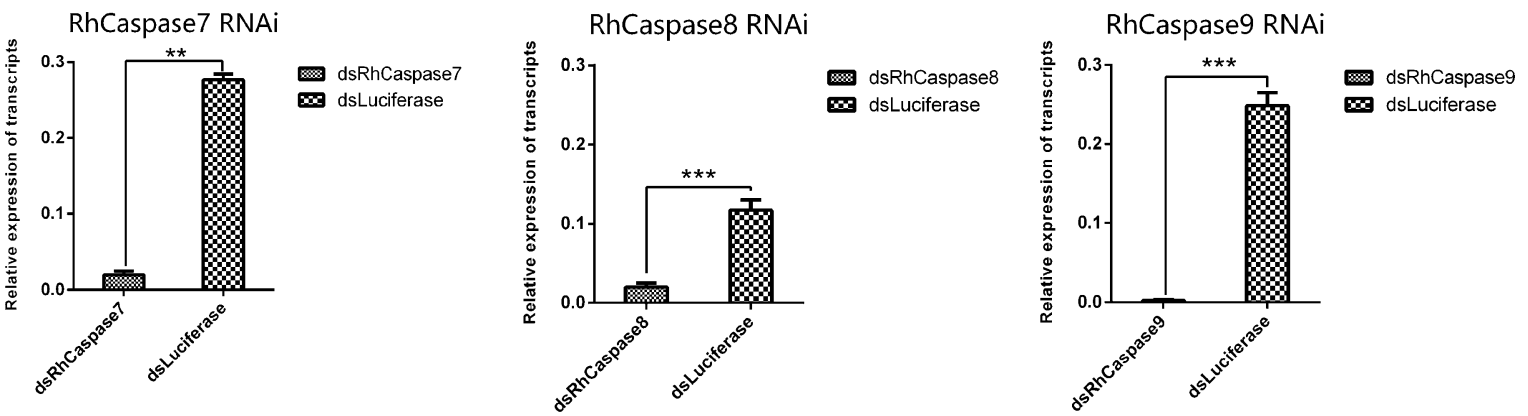

C
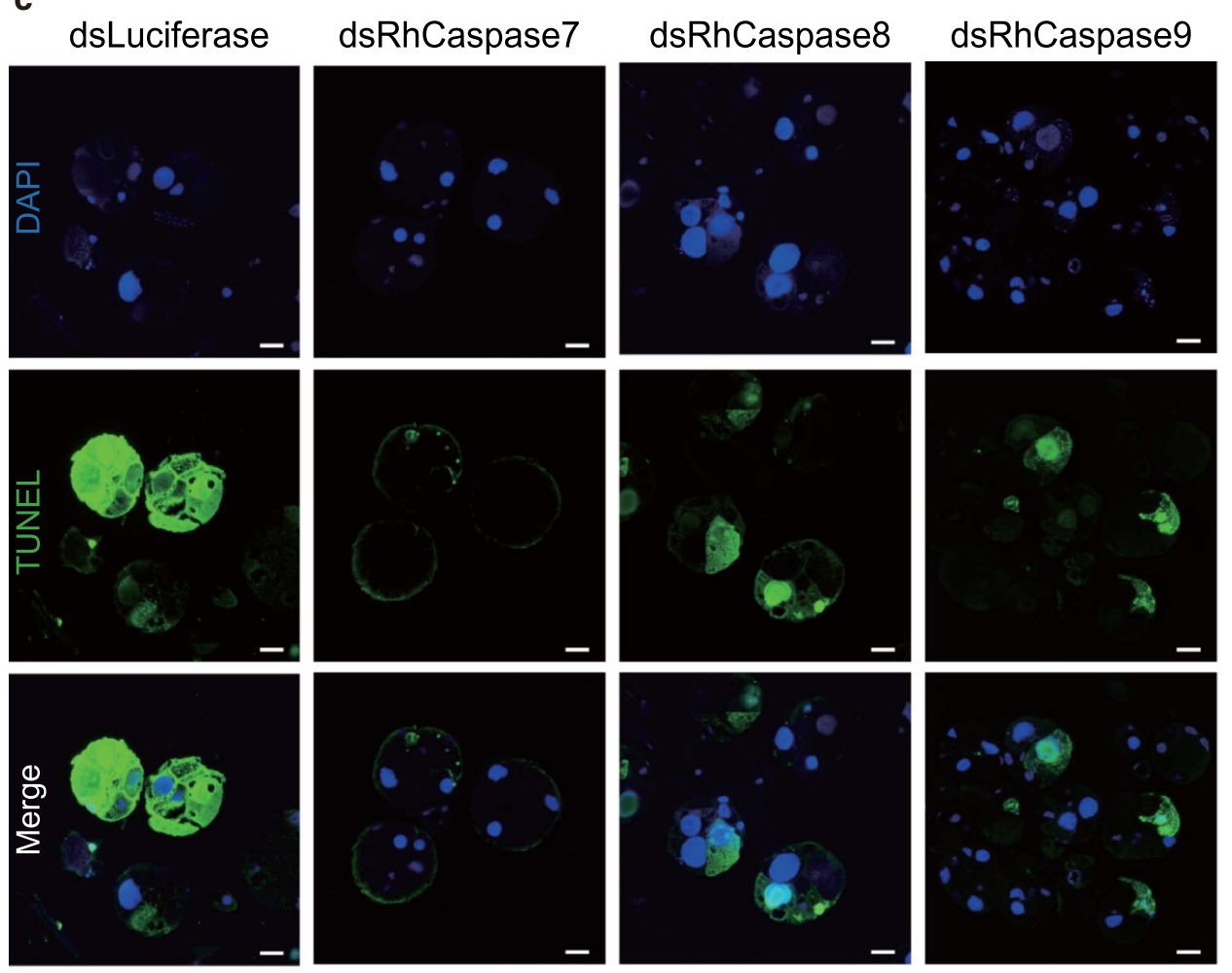
Table 1 Effect of knocking-down RhCaspases7, 8 and 9 on tick feeding behavior

\begin{tabular}{lllll}
\hline Test group & dsRhCaspase 7 & dsRhCaspase 8 & dsRhCaspase 9 & dsLuciferase \\
\hline Attachment rate at 48 $\mathrm{h}(\%)$ & $88.22 \pm 0.906$ & $85.91 \pm 1.256$ & $87.56 \pm 0.957$ & $87.70 \pm 0.690$ \\
Engorgement rate (\%) & $77.12 \pm 2.692$ & $73.157 \pm 3.334$ & $65.236 \pm 4.369$ & $75.79 \pm 3.255$ \\
Engorged tick weight $(\mathrm{mg})$ & $377.1 \pm 89.63$ & $330.157 \pm 40.158$ & $220.157 \pm 50.698$ & $382.1 \pm 57.92$ \\
\hline
\end{tabular}

\section{Discussion}

Tick salivary glands undergo degeneration after engorgement $[4,53,54]$, and apoptosis has a role in this process [13, 14, 33]. Rhipicephalus haemaphysaloides, 1897 (Ixodida: Ixodidae) is a three-host hard tick widely distributed in China [55] and has been reported to be a vector of several pathogens [55-57]. We used an RNA-Seq approach to analyze gene expression differences in unfed and engorged $R$. haemaphysaloides salivary glands. The transcription level of apoptosisrelated genes increased as a consequence of feeding. In addition, we found that apoptosis is involved in salivary gland degeneration.

Caspase genes were differently expressed in the salivary glands of unfed and engorged females. This was consistent with morphological $[58,59]$ and functional [5, 60] effects that occur in tick salivary glands during feeding. Many genes are activated by the feeding process and about $40 \%$ are upregulated. The expression of 40 apoptosis-related genes was affected by the feeding process. The majority of these apoptosis-related genes were upregulated, suggesting a relationship between the feeding process, salivary gland degeneration, and apoptosis.

Compared to other protein families, differentiation of the caspases is complex, since different species have

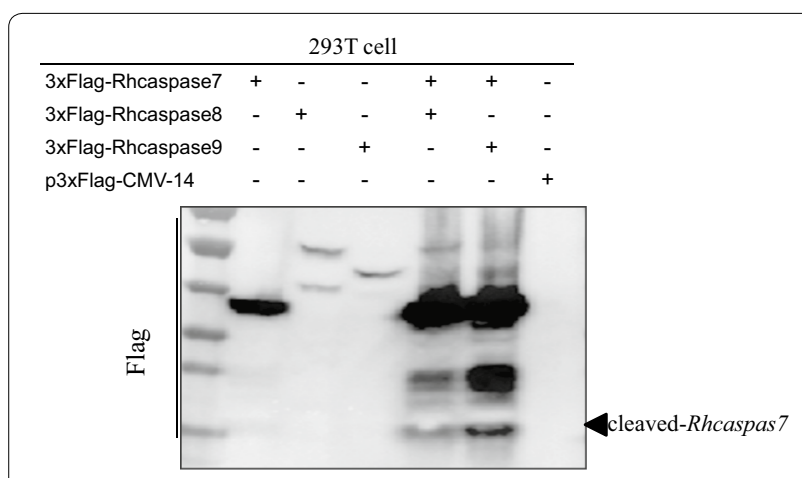

Fig. 8 Western blot analysis of the interaction between RhCaspases 7, 8 and 9 by co-transfection into HEK-293T cells. Lane 1-3: cells transfected individually with p3xFlag-CMV-14-RhCaspase 7, 8 and 9 genes; Lane 4: cells co-transfected with p3×Flag-CMV-14-RhCaspase 7 and p3 $\times$ Flag-CMV-14-RhCaspase 8; Lane 5: cells co-transfected with $\mathrm{p} 3 \times$ Flag-CMV-14-RhCaspase 7 and p3 $\times$ Flag-CMV-14-RhCaspase 9; Lane 6: cells co-transfected with p3×Flag-CMV-14 several isoforms as well as a different number of genes. Also, caspases lack a standardized nomenclature, resulting in differences in nomenclature among species. However, we were able to identify and characterize three $R$. haemaphysaloides caspases, including two initiators (RhCaspases 8 and 9) and one executioner caspase (RhCaspase 7). Sequence analysis showed that RhCaspases 7, 8 and 9 have a conserved sequence characteristic of caspases. However, the two putative initiators, RhCaspases 8 and 9, do not contain the predicted CARD domain.

Although ticks are evolutionarily distant from insects, the phylogenetic analysis of RhCaspases from a variety of insects and other arthropod species suggests a close relationship with caspase homologs in insect species (Fig. 3c). Sometimes, the variability in the caspase nomenclature and number of caspase isotypes among different species makes it difficult to establish comparative relationships. For example, the Drosophila caspases are a well-studied model but the amino acid sequence of caspase DRONC is typical of caspase-2, but functionally similar to caspase-9 [61, 62]. Tick initiator caspases (RhCaspases 8 and 9) are closer to insect initiator caspases and executioner (RhCaspase 7) is more similar to insect executioner caspases.

RNAi is the most effective method for identifying gene functions in ticks [1]. Therefore, RNAi was used to study the physiological roles of the three identified RhCaspases. RhCaspase 9 silencing produced the most significant phenotype alteration in ticks during bloodfeeding compared to RhCaspase $7, \mathrm{RhCaspase} 8$, and the control groups. The RT-qPCR results showed that RhCaspase 9 maintains a high level of transcription in the midgut during all feeding times and it is the most activated caspase in the salivary gland, as determined by western-blot. These data suggest that the role of RhCaspase 9 is related to blood-feeding. However, the precise biological function of RhCaspase 9 during tick feeding remains unclear. The tick salivary gland rapidly degenerates and disappears within 4 days after engorgement $[53,63]$. TUNEL staining has been used to evaluate the rate of DNA fragmentation in degenerated tick salivary glands [13, 14]. Compared with the control group, the positive rate of TUNEL staining was significantly reduced by interfering with the RhCaspase 7,8 
and 9 gene expression using RNAi. Nevertheless, the dsRNAs for RhCaspases 7, 8 and 9 did not completely reduce the degeneration of tick salivary glands. This was expected, since there is redundancy in apoptosis pathways and other types of programmed cell death. In $D$. melanogaster, inhibiting caspase genes or the atg8a gene (autophagy related gene), is not sufficient to prevent salivary gland degradation. Only the simultaneous inhibition of both apoptotic and autophagic pathways impedes salivary gland histolysis [64].

Co-transfection of the cells with plasmids containing caspase genes was used to study the interaction between the different RhCaspases. Although co-transfection is a standard method used to analyze protein-protein interaction events in vitro [65], there are relatively few reports of this type of study in ticks. RhCaspase 7 is an executioner caspase, and RhCaspase 8 and 9 are initiator caspases $[62,66,67]$.

\section{Conclusions}

Three tick caspase molecules were identified in ticks and the roles of these caspases in tick physiology were studied. Our findings provide a basis for advanced studies on tick apoptosis. The knockdown of RhCaspase 9 in vivo inhibited blood-feeding of the tick, demonstrating that RhCaspase9 has the potential to become a candidate vaccine molecule.

\section{Supplementary information}

Supplementary information accompanies this paper at https://doi. org/10.1186/s13071-020-04164-5.

Additional file 1: Table S1. Primers for Rhipicephalus haemaphysaloides caspase genes. Table S2. Primers used for quantitative real-time polymerase chain reactions (qPCRs) of Rhipicephalus haemaphysaloides caspase genes. Table S3. Primers for Rhipicephalus haemaphysaloides caspase ORF cloning *. Table S4. Primers for RNAi of Rhipicephalus haemaphysaloides caspase genes.

Additional file 2: Figure S1. GO terms enrichment analysis for unfed and engorged tick salivary glands. Figure S2. Pheatmap-plot for unfed and engorged tick salivary glands. Figure $\mathbf{S 3}$. Phylogenetic tree of $R$. haemaphysaloides caspases compared to the caspases of Drosophila melanogaster. Bootstrap values of 500 simulations are shown at the branches.

Additional file 3: Alignment S1. Sequences of unigenes (FASTA format).

Additional file 4: Dataset S1. Differentially expressed genes in salivary glands of unfed and engorged ticks.

\section{Abbreviations}

TBPs: tick-borne pathogens; PCD: programmed cell death; PCAb: polyclonal antibodies; RT-qPCR: reverse transcription quantitative PCR; PBS: phosphatebuffered saline; TBS: Tris-buffered saline; GO: Gene: Gene Ontology; KOG: euKaryotic Orthologous Groups; KEGG: Kyoto Encyclopedia of Genes and Genomes; SDS-PAGE: sodium dodecyl sulfate polyacrylamide gel electrophoresis; IPTG: isopropyl $\beta$-D-1-thiogalactopyranoside; DAPI: 4', 6'-diamidino2-phenylindole; TUNEL: terminal deoxynucleotidyl transferase dUTP nick-end labeling.

\section{Acknowledgments}

We thank LetPub (www.letpub.com) for its linguistic assistance during the preparation of this manuscript.

\section{Authors' contributions}

YW conceived the study, performed the statistical analyses and participated in the dynamic detection of the target genes. YW drafted the manuscript. XY and $\mathrm{HZ}$ participated in the design of the study and the interpretation of the data. SH and YM participated in the animal experiments. JZ and IDSVJ participated in the design of the study and helped to draft the manuscript. All authors read and approved the final manuscript.

\section{Funding}

This work was supported by grants from The Agricultural Science and Technology Innovation Program (ASTIP) of China (CAAS-XTCX2016011-02).

\section{Availability of data and materials}

The datasets supporting the conclusions of this article are included within the article and its additional files.

\section{Ethics approval and consent to participate}

The treatment of rabbits and mice was approved by the Institutional Animal Care and Use Committee of the Shanghai Veterinary Research Institute (IACUC approval number: SHVRI-mo-20180306-008; SHVRI-ra-20180415-04), and authorized by the Animal Ethical Committee of Shanghai Veterinary Research Institute.

\section{Consent for publication}

Not applicable.

\section{Competing interests}

The authors declare that they have no competing interests.

\section{Author details}

${ }^{1}$ Key Laboratory of Animal Parasitology of Ministry of Agriculture, Shanghai Veterinary Research Institute, Chinese Academy of Agricultural Sciences, Shanghai 200241, China. ${ }^{2}$ Centro de Biotecnologia, Universidade Federal do Rio Grande do Sul, Porto Alegre, RS, Brazil.

Received: 17 February 2020 Accepted: 1 June 2020 Published online: 05 June 2020

\section{References}

1. de la Fuente J, Kocan KM, Almazan C, Blouin EF. Targeting the tick-pathogen interface for novel control strategies. Front Biosci. 2008;13:6947-56.

2. Ferreri L, Giacobini M, Bajardi P, Bertolotti L, Bolzoni L, Tagliapietra V, et al. Pattern of tick aggregation on mice: larger than expected distribution tail enhances the spread of tick-borne pathogens. PLoS Comput Biol. 2014;10:e1003931.

3. Dantas-Torres F, Chomel BB, Otranto D. Ticks and tick-borne diseases: a one health perspective. Trends Parasitol. 2012;28:437-46.

4. Sonenshine DE. Biology of ticks. New York: Oxford University Press; 1991

5. Simo L, Kazimirova M, Richardson J, Bonnet SI. The essential role of tick salivary glands and saliva in tick feeding and pathogen transmission. Front Cell Infect Microbiol. 2017;7:281.

6. Kazimirova M, Stibraniova I. Tick salivary compounds: their role in modulation of host defences and pathogen transmission. Front Cell Infect Microbiol. 2013;3:43.

7. Bayles KW. Bacterial programmed cell death: making sense of a paradox. Nat Rev Microbiol. 2014;12:63-9.

8. Zheng W, Rasmussen U, Zheng S, Bao X, Chen B, Gao Y, et al. Multiple modes of cell death discovered in a prokaryotic (cyanobacterial) endosymbiont. PLoS ONE. 2013;8:e66147.

9. Koonin EV, Aravind L. Origin and evolution of eukaryotic apoptosis: the bacterial connection. Cell Death Differ. 2002;9:394-404.

10. Jacobson MD, Weil M, Raff MC. Programmed cell death in animal development. Cell. 1997;88:347-54. 
11. Maghsoudi N, Zakeri Z, Lockshin RA. Programmed cell death and apoptosis - where it came from and where it is going: from Elie Metchnikoff to the control of caspases. Exp Oncol. 2012;34:146-52.

12. Bursch W, Ellinger A, Gerner C, Frohwein U, Schulte-Hermann R. Programmed cell death (PCD). Apoptosis, autophagic PCD, or others? Ann N Y Acad Sci. 2000;926:1-12.

13. L'Amoreaux WJ, Junaid L, Trevidi S. Morphological evidence that salivary gland degeneration in the American dog tick, Dermacentor variabilis (Say), involves programmed cell death. Tissue Cell. 2003;35:95-9.

14. Freitas DR, Rosa RM, Moura DJ, Seitz AL, Colodel EM, Driemeier D, et al. Cell death during preoviposition period in Boophilus microplus tick. Vet Parasitol. 2007; 144:321-7.

15. Wolf BB, Green DR. Suicidal tendencies: apoptotic cell death by caspase family proteinases. J Biol Chem. 1999;274:20049-52.

16. Thornberry NA, Lazebnik Y. Caspases: enemies within. Science. 1998;281:1312-6.

17. Rai NK, Tripathi K, Sharma D, Shukla VK. Apoptosis: a basic physiologic process in wound healing. Int J Low Extrem Wounds. 2005:4:138-44.

18. Cohen GM. Caspases: the executioners of apoptosis. Biochem J. 1997;326:1-16.

19. Elmore S. Apoptosis: a review of programmed cell death. Toxicol Pathol. 2007;35:495-516.

20. Bouchier-Hayes L, Martin SJ. CARD games in apoptosis and immunity. EMBO Rep. 2002;3:616-21.

21. Mcllwain DR, Berger T, Mak TW. Caspase functions in cell death and disease. Cold Spring Harb Perspect Biol. 2015;7:a008656.

22. Shalini S, Dorstyn L, Dawar S, Kumar S. Old, new and emerging functions of caspases. Cell Death Differ. 2015;22:526-39.

23. Scott AM, Saleh M. The inflammatory caspases: guardians against infections and sepsis. Cell Death Differ. 2007;14:23-31.

24. Shi J, Zhao Y, Wang Y, Gao W, Ding J, Li P, et al. Inflammatory caspases are innate immune receptors for intracellular LPS. Nature. 2014;514:187-92.

25. Zermati Y, Garrido C, Amsellem S, Fishelson S, Bouscary D, Valensi F, et al. Caspase activation is required for terminal erythroid differentiation. J Exp Med. 2001;193:247-54.

26. Ishizaki Y, Jacobson MD, Raff MC. A role for caspases in lens fiber differentiation. J Cell Biol. 1998;140:153-8.

27. Fan $W$, Dai $Y, X u H$, Zhu X, Cai $P$, Wang $L$, et al. Caspase-3 modulates regenerative response after stroke. Stem Cells. 2014;32:473-86.

28. Fernando P, Kelly JF, Balazsi K, Slack RS, Megeney LA. Caspase 3 activity is required for skeletal muscle differentiation. Proc Natl Acad Sci USA. 2002;99:11025-30.

29. Xu HL, Xu WH, Cai Q, Feng M, Long J, Zheng W, et al. Polymorphisms and haplotypes in the caspase-3, caspase-7, and caspase-8 genes and risk for endometrial cancer: a population-based, case-control study in a Chinese population. Cancer Epidemiol Biomarkers Prev. 2009;18:2114-22.

30. Ho LH, Taylor R, Dorstyn L, Cakouros D, Bouillet P, Kumar S. A tumor suppressor function for caspase-2. Proc Natl Acad Sci USA. 2009;106:5336-41.

31. Shalini S, Puccini J, Wilson CH, Finnie J, Dorstyn L, Kumar S. Caspase-2 protects against oxidative stress in vivo. Oncogene. 2015;34:4995-5002.

32. Zhang Y, Padalecki SS, Chaudhuri AR, De Waal E, Goins BA, Grubbs B, et al. Caspase-2 deficiency enhances aging-related traits in mice. Mech Ageing Dev. 2007;128:213-21

33. Yu X, Zhou Y, Cao J, Zhang H, Gong H, Zhou J. Caspase-1 participates in apoptosis of salivary glands in Rhipicephalus haemaphysaloides. Parasit Vectors. 2017;10:225.

34. Yu X, Gong H, Zhou Y, Zhang H, Cao J, Zhou J. Differential sialotranscriptomes of unfed and fed Rhipicephalus haemaphysaloides, with particular regard to differentially expressed genes of cysteine proteases. Parasit Vectors. 2015;8:597.

35. Tanaka M, Liao M, Zhou J, Nishikawa Y, Xuan X, Fujisaki K. Molecular cloning of two caspase-like genes from the hard tick Haemaphysalis longicornis. J Vet Med Sci. 2007;69:85-90.

36. Ayllon N, Villar M, Galindo RC, Kocan KM, Sima R, Lopez JA, et al. Systems biology of tissue-specific response to Anaplasma phagocytophilum reveals differentiated apoptosis in the tick vector Ixodes scapularis. PLoS Genet. 2015;11:e1005120.

37. Grabherr MG, Haas BJ, Yassour M, Levin JZ, Thompson DA, Amit I, et al. Full-length transcriptome assembly from RNA-seq data without a reference genome. Nat Biotechnol. 2011;29:644-52.
38. Pertea G, Huang X, Liang F, Antonescu V, Sultana R, Karamycheva S, et al. TIGR gene indices clustering tools (TGICL): a software system for fast clustering of large EST datasets. Bioinformatics. 2003;19:651-2.

39. Gene Ontology C. The gene ontology project in 2008. Nucleic Acids Res. 2008:36:D440-4

40. Tatusov RL, Galperin MY, Natale DA, Koonin EV. The COG database: a tool for genome-scale analysis of protein functions and evolution. Nucleic Acids Res. 2000;28:33-6.

41. Kanehisa M, Goto S, Kawashima S, Okuno Y, Hattori M. The KEGG resource for deciphering the genome. Nucleic Acids Res. 2004;32:D277-80.

42. Zdobnov EM, Apweiler R. InterProScan - an integration platform for the signature-recognition methods in InterPro. Bioinformatics. 2001;17:847-8.

43. Cruz LM, Trefflich S, Weiss VA, Castro MAA. Protein function prediction. Methods Mol Biol. 2017:1654:55-75.

44. Artimo P, Jonnalagedda M, Arnold K, Baratin D, Csardi G, de Castro E, et al. ExPASy: SIB bioinformatics resource portal. Nucleic Acids Res. 2012;40:W597-603.

45. Edgar RC. MUSCLE: multiple sequence alignment with high accuracy and high throughput. Nucleic Acids Res. 2004;32:1792-7.

46. Kumar S, Stecher G, Li M, Knyaz C, Tamura K. MEGA X: Molecular evolutionary genetics analysis across computing platforms. Mol Biol Evol. 2018;35:1547-9.

47. Grinshpon RD, Williford A, Titus-McQuillan J, Clay Clark A. The CaspBase: a curated database for evolutionary biochemical studies of caspase functional divergence and ancestral sequence inference. Protein Sci. 2018;27:1857-70

48. Nijhof AM, Balk JA, Postigo M, Jongejan F. Selection of reference genes for quantitative RT-PCR studies in Rhipicephalus (Boophilus) microplus and Rhipicephalus appendiculatus ticks and determination of the expression profile of Bm86. BMC Mol Biol. 2009;10:112.

49. Livak KJ, Schmittgen TD. Analysis of relative gene expression data using real-time quantitative PCR and the 2(-Delta Delta C(T)) method. Methods. 2001;25:402-8.

50. Ginzinger DG. Gene quantification using real-time quantitative PCR: an emerging technology hits the mainstream. Exp Hematol. 2002;30:503-12.

51. Suganuma I, Ushiyama T, Yamada H, Iwamoto A, Kobayashi M, Ikeda M. Cloning and characterization of a dronc homologue in the silkworm, Bombyx mori. Insect Biochem Mol Biol. 2011;41:909-21.

52. Huang N, Civciristov S, Hawkins CJ, Clem RJ. SfDronc, an initiator caspase involved in apoptosis in the fall armyworm Spodoptera frugiperda. Insect Biochem Mol Biol. 2013;43:444-54.

53. Francischetti IM, Sa-Nunes A, Mans BJ, Santos IM, Ribeiro JM. The role of saliva in tick feeding. Front Biosci (Landmark Ed). 2009;14:2051-88.

54. Sauer JR, McSwain JL, Bowman AS, Essenberg RC. Tick salivary gland physiology. Annu Rev Entomol. 1995;40:245-67.

55. Chen Z, Yang X, Bu F, Yang X, Yang X, Liu J. Ticks (Acari: Ixodoidea: Argasidae, Ixodidae) of China. Exp Appl Acarol. 2010;51:393-404.

56. Yu Z, Wang H, Wang T, Sun W, Yang X, Liu J. Tick-borne pathogens and the vector potential of ticks in China. Parasit Vectors. 2015;8:24.

57. Zhang J, Liu Q, Wang D, Li W, Beugnet F, Zhou J. Epidemiological survey of ticks and tick-borne pathogens in pet dogs in south-eastern China. Parasite. 2017;24:35.

58. Barker DM, Ownby CL, Krolak JM, Claypool PL, Sauer JR. The effects of attachment, feeding, and mating on the morphology of the type I alveolus of salivary glands of the lone star tick, Amblyomma americanum (L.). J Parasitol. 1984;70:99-113.

59. Aljamali MN, Ramakrishnan VG, Weng H, Tucker JS, Sauer JR, Essenberg RC. Microarray analysis of gene expression changes in feeding female and male lone star ticks, Amblyomma americanum (L). Arch Insect Biochem Physiol. 2009;71:236-53.

60. Tirloni L, Reck J, Terra RM, Martins JR, Mulenga A, Sherman NE, et al. Proteomic analysis of cattle tick Rhipicephalus (Boophilus) microplus saliva: a comparison between partially and fully engorged females. PLOS ONE. 2014;9:e94831.

61. Kumar S. Caspase function in programmed cell death. Cell Death Differ. 2007;14:32-43.

62. Kumar S, Doumanis J. The fly caspases. Cell Death Differ. 2000;7:1039-44.

63. Umemiya-Shirafuji R, Galay RL, Maeda H, Kawano S, Tanaka T, Fukumoto $\mathrm{S}$, et al. Expression analysis of autophagy-related genes in the hard tick Haemaphysalis longicornis. Vet Parasitol. 2014;201:169-75. 
64. Accorsi A, Zibaee A, Malagoli D. The multifaceted activity of insect caspases. J Insect Physiol. 2015;76:17-23.

65. Lin JS, Lai EM. Protein-protein interactions: co-immunoprecipitation. Methods Mol Biol. 2017;1615:211-9.

66. Lamkanfi M, Declercq W, Kalai M, Saelens X, Vandenabeele P. Alice in caspase land. A phylogenetic analysis of caspases from worm to man. Cell Death Differ. 2002;9:358-61.
67. Courtiade J, Pauchet Y, Vogel H, Heckel DG. A comprehensive characterization of the caspase gene family in insects from the order Lepidoptera. BMC Genomics. 2011;12:357.

\section{Publisher's Note}

Springer Nature remains neutral with regard to jurisdictional claims in published maps and institutional affiliations.
Ready to submit your research? Choose BMC and benefit from:

- fast, convenient online submission

- thorough peer review by experienced researchers in your field

- rapid publication on acceptance

- support for research data, including large and complex data types

- gold Open Access which fosters wider collaboration and increased citations

- maximum visibility for your research: over $100 \mathrm{M}$ website views per year

At BMC, research is always in progress.

Learn more biomedcentral.com/submissions 